\title{
Electromagnetic solitary pulses in a magnetized electron-positron plasma
}

\author{
P K Shukla, B Eliasson and Lennart Stenflo
}

\section{Linköping University Post Print}

N.B.: When citing this work, cite the original article.

Original Publication:

P K Shukla, B Eliasson and Lennart Stenflo, Electromagnetic solitary pulses in a magnetized electron-positron plasma, 2011, Physical Review E. Statistical, Nonlinear, and Soft Matter Physics, (84), 3, 037401.

http://dx.doi.org/10.1103/PhysRevE.84.037401

Copyright: American Physical Society

http://www.aps.org/

Postprint available at: Linköping University Electronic Press

http://urn.kb.se/resolve?urn=urn:nbn:se:liu:diva-70744 


\title{
Electromagnetic solitary pulses in a magnetized electron-positron plasma
}

\author{
P. K. Shukla, ${ }^{1}$ B. Eliasson, ${ }^{2}$ and L. Stenflo ${ }^{3}$ \\ ${ }^{1}$ RUB International Chair, International Centre for Advanced Studies in Physical Sciences, Fakultät für Physik und Astronomie, \\ Ruhr-Universität Bochum, D-44780 Bochum, Germany \\ ${ }^{2}$ Institut für Theoretische Physik, Fakultät für Physik und Astronomie, Ruhr-Universität Bochum, D-44780 Bochum, Germany \\ ${ }^{3}$ Department of Physics, Linköping University, SE-58183 Linköping, Sweden
}

(Received 31 May 2011; revised manuscript received 20 July 2011; published 1 September 2011)

\begin{abstract}
A theory for large amplitude compressional electromagnetic solitary pulses in a magnetized electron-positron $(e-p)$ plasma is presented. The pulses, which propagate perpendicular to the external magnetic field, are associated with the compression of the plasma density and the wave magnetic field. Here the solitary wave magnetic field pressure provides the restoring force, while the inertia comes from the equal mass electrons and positrons. The solitary pulses are formed due to a balance between the compressional wave dispersion arising from the curl of the inertial forces in Faraday's law and the nonlinearities associated with the divergence of the electron and positron fluxes, the nonlinear Lorentz forces, the advection of the $e$ - $p$ fluids, and the nonlinear plasma current densities. The compressional solitary pulses can exist in a well-defined speed range above the Alfvén speed. They can be associated with localized electromagnetic field excitations in magnetized laboratory and space plasmas composed of electrons and positrons.
\end{abstract}

DOI: 10.1103/PhysRevE.84.037401

PACS number(s): 52.27.Ep, 52.25.Xz, 52.35.Mw, 52.35.Sb

\section{INTRODUCTION}

Electron-positron $(e-p)$ plasmas are found in the early universe [1,2], in astrophysical objects (e.g., pulsars [3], supernova remnants, and active galactic nuclei $[4,5])$, in $\gamma$-ray bursts [6], and at the center of the Milky Way galaxy [7]. In such physical systems, the $e-p$ pairs can be created by collisions between particles that are accelerated by electromagnetic and electrostatic waves and/or by gravitational forces. In pulsar environments, there is also a possibility of pair creation via high-energy curvature radiation photons that are triggered by charged particles streaming along the curved magnetic field $[8,9]$, with a resulting collection of positrons at the polar caps of the pulsar [10-12]. High-energy laser-plasma interactions and fusion devices also constitute a source of $e$ - $p$ plasmas (also referred to as pair plasma). Intense laser-plasma interaction experiments have reported the production of $\mathrm{MeV}$ electrons and conclusive evidence of positron production [13-16] via electron collisions [17]. Positrons have also been created in postdisruption plasmas in large tokamaks [18] through collisions between $\mathrm{MeV}$ electrons and thermal particles. The progress in the production of positron plasmas of the past two decades makes it possible to consider laboratory experiments on $e-p$ plasmas [19-21].

The pair plasmas are composed of electrons and positrons of equal mass and opposite charges. Due to the equal mass of the pairs, there are fewer spatial and temporal scales on which collective effects (e.g., electrostatic and electromagnetic waves as well as their instabilities, coherent nonlinear structures, etc.) arise. For example, Iwamoto [22] presented an elegant description of the linear modes in a nonrelativistic pair plasma that is magnetized. Zank and Greaves [23] then discussed the linear properties of various electrostatic and electromagnetic waves in unmagnetized and magnetized pair plasmas, in addition to discussing the two-stream instability and nonenvelope solitary wave solutions. It should be noted that the magneticfield-aligned circularly polarized electromagnetic (CPEM) waves and elliptically polarized extraordinary (EPEO) waves propagating across the external magnetic field direction have the same frequencies [24-26] in the cold $e-p$ magnetoplasma. Several authors [27-30] have investigated the properties of magnetic-field-aligned nonlinear Alfvén-like waves in a relativistic magnetized pair plasma. A review of numerous linear and nonlinear waves in unmagnetized and magnetized pair plasmas can be found in Ref. [31]. It turns out that studies of collective plasma wave phenomena are of great importance in connection with energization of electrons and positrons in a magnetized pair plasma.

In this Brief Report, we present an investigation of largeamplitude compressional electromagnetic solitary pulses that are propagating across the external magnetic field in a pair plasma. In our quasineutral pair plasma, the dynamics of the compressional electromagnetic solitary waves is governed by the continuity and momentum equations, as well as Ampère's and Faraday's laws. In a stationary frame with a constant speed, the governing equations can be reduced to a pair of equations in which the compressional magnetic field and the plasma density are nonlinearly coupled, as shown in Sec. II. In Sec. III, we carry out a numerical analysis of the nonlinear equations which reveals that the solitary electromagnetic pulses are composed of density and magnetic field humps and have some similarities with the compressional Alfvénic solitons in a cold electronion plasma [32]. The properties and existence criteria of the solitary electromagnetic pulses are discussed, and examples of profiles of the solitary pulses are displayed graphically. It is found that a stronger localization of solitary pulses occurs in a cold pair magnetoplasma. Section IV contains a summary and possible implications of our work for astrophysical and laboratory pair plasmas.

\section{THE NONLINEAR MODEL}

Let us consider the nonlinear propagation of large amplitude compressional electromagnetic waves across the external magnetic field $B_{0} \hat{\mathbf{z}}$, where $B_{0}$ is the strength of the external 
magnetic field and $\hat{\mathbf{z}}$ the unit vector along the $z$ axis in a Cartesian coordinate system, in a quasineutral electronpositron plasma. In the latter, we have $n_{e}=n_{p} \equiv n$, where $n_{e}$ and $n_{p}$ are the number densities of the electrons and positrons. For one-dimensional electromagnetic wave propagation along the $x$ axis, the continuity equations for the electrons and positrons predict that the $x$ components of the electron and positron fluid velocities must be equal, viz., $v_{e x}=v_{p x} \equiv v_{x}$. Hence the continuity equation for both the electrons and positrons reads

$$
\frac{\partial n}{\partial t}+\frac{\partial\left(n v_{x}\right)}{\partial x}=0 .
$$

Furthermore, by using $n$ and $v_{x}$ in the $x$ components of the electron and positron momentum equations, we have

$$
m\left(\frac{\partial}{\partial t}+v_{x} \frac{\partial}{\partial x}\right) v_{x}=-e E_{x}-\frac{e}{c} v_{e y} B-\frac{k_{B} T}{n n_{0}^{2}} \frac{\partial n^{3}}{\partial x}
$$

and

$$
m\left(\frac{\partial}{\partial t}+v_{x} \frac{\partial}{\partial x}\right) v_{x}=e E_{x}+\frac{e}{c} v_{p y} B-\frac{k_{B} T}{n n_{0}^{2}} \frac{\partial n^{3}}{\partial x},
$$

where $m$ is the electron or positron mass, $e$ the magnitude of the electron charge, $E_{x}$ the $x$ component of the wave electric field, $B$ the sum of the ambient and compressional (along the $z$ axis) magnetic fields, $c$ the speed of light in vacuum, $k_{B}$ the Boltzmann constant, $T$ the common constant temperature of the electrons and positrons, and $n_{0}$ the unperturbed plasma number density. The last term in the right-hand side of Eqs. (2) and (3) corresponds to the adiabatic equation of state for the electron and positron pressure $P=P_{0}\left(n / n_{0}\right)^{\gamma}$, where $P_{0}=n_{0} k_{B} T$ is the equilibrium pressure at $n=n_{0}$, where $\gamma=C_{P} / C_{V}=(N+2) / N$ is the ratio between specific heats and $N$ is the number of degrees of freedom in the system. We have chosen $N=1$ for one-dimensional compression.

Eliminating $E_{x}$ from Eq. (2) by using Eq. (3) we have

$$
2 m\left(\frac{\partial}{\partial t}+v_{x} \frac{\partial}{\partial x}\right) v_{x}=\frac{e}{c}\left(v_{p y}-v_{e y}\right) B-6 \frac{k_{B} T}{n_{0}^{2}} n \frac{\partial n}{\partial x},
$$

which yields

$$
\left(\frac{\partial}{\partial t}+v_{x} \frac{\partial}{\partial x}\right) v_{x}=-\frac{B}{8 \pi m n} \frac{\partial B}{\partial x}-3 \frac{k_{B} T}{m n_{0}^{2}} n \frac{\partial n}{\partial x},
$$

where we have used Ampère's law,

$$
\frac{\partial B}{\partial x}=-\frac{4 \pi e n}{c}\left(v_{p y}-v_{e y}\right)
$$

which is valid for compressional electromagnetic waves with phase velocities much smaller than $c$.

For our purposes, Faraday's law reads

$$
\frac{\partial B}{\partial t}=-c \frac{\partial E_{y}}{\partial x}
$$

where $E_{y}$ is the $y$ component of the wave electric field.

The $y$ components of the electron and positron momentum equations are

$$
m\left(\frac{\partial}{\partial t}+v_{x} \frac{\partial}{\partial x}\right) v_{e y}=-e E_{y}+\frac{e}{c} v_{x} B
$$

and

$$
m\left(\frac{\partial}{\partial t}+v_{x} \frac{\partial}{\partial x}\right) v_{p y}=e E_{y}-\frac{e}{c} v_{x} B .
$$

Subtracting Eq. (8) from Eq. (9), we have

$$
m\left(\frac{\partial}{\partial t}+v_{x} \frac{\partial}{\partial x}\right)\left(v_{p y}-v_{e y}\right)=2 e E_{y}-2 \frac{e}{c} v_{x} B,
$$

which, by using Eq. (6), gives

$$
E_{y}=\frac{v_{x} B}{c}-\frac{m c}{8 \pi e^{2}}\left(\frac{\partial}{\partial t}+v_{x} \frac{\partial}{\partial x}\right)\left(\frac{1}{n} \frac{\partial B}{\partial x}\right) .
$$

From Eqs. (7) and (11) we then obtain

$$
\frac{\partial B}{\partial t}+\frac{\partial\left(v_{x} B\right)}{\partial x}-\frac{m c^{2}}{8 \pi e^{2}} \frac{\partial}{\partial x}\left(\frac{\partial}{\partial t}+v_{x} \frac{\partial}{\partial x}\right)\left(\frac{1}{n} \frac{\partial B}{\partial x}\right)=0 .
$$

Equations (1), (5), and (12) are the governing nonlinear equations for the compressional electromagnetic waves in a warm $e$ - $p$ magnetoplasma.

Letting $n=n_{0}+n_{1}$ and $B=B_{0}+B_{1}$, where $n_{1} \ll n_{0}$ and $B_{1} \ll B_{0}$, we first linearize the system of Eqs. (1), (5), and (12) and combine the resultant equations to obtain the wave equation

$$
\left[\left(1-\lambda_{e}^{2} \frac{\partial^{2}}{\partial x^{2}}\right)\left(\frac{\partial^{2}}{\partial t^{2}}-3 V_{T}^{2} \frac{\partial^{2}}{\partial x^{2}}\right)-C_{A}^{2} \frac{\partial^{2}}{\partial x^{2}}\right] B_{1}=0
$$

where $\lambda_{e}=c / \omega_{p e}$ is the electron/positron skin depth, $\omega_{p e}=\left(8 \pi n_{0} e^{2} / m\right)^{1 / 2}$ the electron plasma frequency, $V_{T}=$ $\left(k_{B} T / m\right)^{1 / 2}$ the thermal speed, and $C_{A}=B_{0} / \sqrt{8 \pi n_{0} m}$ the Alfvén speed in the $e-p$ plasma.

Assuming that $B_{1}$ is proportional to $\exp (-i \omega t+i k x)$, where $\omega$ and $k$ are the frequency and the wave number, respectively, we next Fourier transform Eq. (13) to obtain

$$
\omega^{2}=3 k^{2} V_{T}^{2}+\frac{k^{2} C_{A}^{2}}{1+k^{2} \lambda_{e}^{2}} .
$$

We note that the frequency of the compressional electromagnetic wave, given by Eq. (14), differs from the frequencies of the CPEM and EPEO waves in a magnetized pair plasma. It should be stressed that the CPEM wave does not accompany a density perturbation, contrary to the EPEO and compressional electromagnetic waves which have density fluctuations.

Let us now investigate the properties of the nonlinear compressional electromagnetic pulses in a warm $e-p$ magnetoplasma. For this purpose, we normalize $n$ by $n_{0}, B$ by $B_{0}$, $v_{x}$ by the Alfvèn speed $C_{A}$, and the time and space variables by $\omega_{c e}^{-1}=m c / e B_{0}$ and $\lambda_{A}=C_{A} / \omega_{c e} \equiv \lambda_{e}$, and we rewrite Eqs. (1), (5), and (12) as

$$
\begin{gathered}
\frac{D n}{d t}+n \frac{\partial v_{x}}{\partial x}=0, \\
\frac{D v_{x}}{d t}+\frac{1}{2 n} \frac{\partial B^{2}}{\partial x}+\frac{3 \beta}{2} \frac{\partial n^{2}}{\partial x}=0,
\end{gathered}
$$

and

$$
\frac{\partial B}{\partial t}+\frac{\partial\left(v_{x} B\right)}{\partial x}-\frac{\partial}{\partial x}\left[\frac{D}{d t}\left(\frac{1}{n} \frac{\partial B}{\partial x}\right)\right]=0,
$$



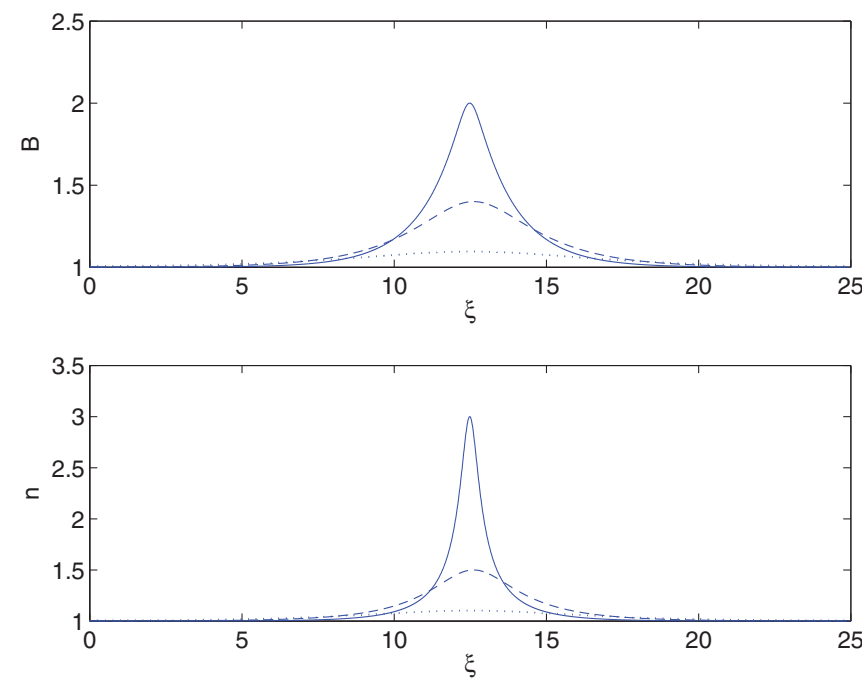

FIG. 1. (Color online) The spatial profiles of the normalized magnetic field $B$ (top panel) and the plasma density $n$ (bottom panel) for $\beta=0$ and Mach numbers $M=1.2$ (dashed curves) and $M=1.5$ (solid curves). The amplitude decreases for finite $\beta$, for example, for $\beta=0.1$ and $M=1.2$ (dotted curves).

where $\quad D / d t=(\partial / \partial t)+v_{x} \partial / \partial x \quad$ and $\quad \beta=V_{T}^{2} / C_{A}^{2} \equiv$ $8 \pi k_{B} n_{0} T / B_{0}^{2}$.

We now seek solitary pulse solutions of Eqs. (15)-(17) in a stationary frame $\xi=x-M t$, where $M=v_{0} / C_{A}$ is the Mach number and $v_{0}$ is the constant propagation speed of the solitary pulses. Thus, from Eqs. (15), (16), and (17) we have, respectively,

$$
\begin{gathered}
v_{x}=M(1-1 / n), \\
M^{2}\left(\frac{1}{n}-1\right)+\frac{1}{2}\left(B^{2}-1\right)+\beta\left(n^{3}-1\right)=0,
\end{gathered}
$$

and

$$
\frac{\partial}{\partial \xi}\left(\frac{1}{n} \frac{\partial B}{\partial \xi}\right)-B+n=0,
$$

where for solitary electromagnetic waves we have imposed the boundary conditions $v_{x}=0, n=1, B=1$, and $\partial B / \partial \xi=0$ at $|\xi|=\infty$.

\section{PROFILES OF SOLITARY PULSES}

Numerical solutions of Eqs. (19) and (20) are displayed in Fig. 1, which shows the profiles of large-amplitude compres- sional electromagnetic solitary pulses for $M=1.2$ and $M=$ 1.5 and for different values of $\beta$. It is seen that both the density and the magnetic field are compressed at the center of the solitary electromagnetic pulse and that the amplitude increases with increasing values of $M$. For finite $\beta$, the amplitudes of the solitary pulses quickly decrease, as can be seen from the dotted curve for $\beta=0.1$ and $M=1.2$. It is found that compressional solitary electromagnetic pulses only exist in the range $\sqrt{1+3 \beta}<M<M_{\max }$, where the amplitude goes to zero as $M \rightarrow \sqrt{1+3 \beta}$. The lower limit of $M$ corresponds to the linear acoustic wave speed at long wavelengths, and hence the solitary pulses are always superacoustic. The maximum speed $M_{\max }$ depends on $\beta$. For $\beta=0$, we find numerically that $M_{\max }=2$, and for $M \rightarrow 2$ we find a maximum value of $B \rightarrow 3$ and a pileup of the density at the center of the solitary pulse in this limit. For finite $\beta$, both the magnetic-field and the plasma-density variations are always finite and decrease for larger values of $\beta$.

\section{SUMMARY AND CONCLUSIONS}

In this Brief Report, we have investigated the properties of large-amplitude compressional electromagnetic pulses propagating perpendicular to the ambient magnetic field in a pair plasma composed of electrons and positrons. It is found that solitary electromagnetic pulses can exist within a definite velocity range between the Alfvénic speed and twice the Alfvénic speed in our pair plasma. The solitary electromagnetic pulses are associated with an enhancement of both the plasma density and the magnetic field, and they experience a sharp pileup of the density for speeds approaching twice the Alfvénic speed. Furthermore, it is found that a strong localization of pulses occurs in a cold pair plasma. In conclusion, we stress that the present solitary pulses can be associated with large-amplitude compressional electromagnetic disturbances that can occur in nonrelativistic magnetized pair plasmas, such as those in active galactic nuclei and in laboratory experiments. Finally, we have to explore new aspects of nonlinear plasma waves and their role with regard to electron and positron acceleration in inertial confinement fusion plasmas and in small-scale laboratory discharges that are composed of magnetized electrons and positrons.

\section{ACKNOWLEDGMENTS}

This research was supported by the Deutsche Forschungsgemeinschaft (DFG) through Project SH21/3-1 of Research Unit 1048.
[1] W. Misner, K. S. Thorne, and J. A. Wheeler, Gravitation (Freeman, San Francisco, 1973), p. 763.

[2] G. W. Gibbons, S. W. Hawking, and S. Siklos, The Very Early Universe (Cambridge University Press, Cambridge, UK, 1983).

[3] R. J. Manchester and J. H. Taylor, Pulsars (Freeman, San Francisco, 1977).

[4] M. C. Begelman, R. D. Blandford, and M. D. Rees, Rev. Mod. Phys. 56, 255 (1984).
[5] H. R. Miller and P. J. Witta, in Active Galactic Nuclei (Springer, Berlin, 1987), p. 202.

[6] T. Piran, Phys. Rep. 314, 575 (1999); Rev. Mod. Phys. 76, 1143 (2004).

[7] M. L. Burns, in Positron-Electrons Pairs in Astrophysics, edited by M. L. Burns, A. K. Harding, and R. Ramaty (American Institute of Physics, New York, 1983).

[8] V. L. Ginzburg, Sov. Phys. Usp. 14, 83 (1971). 
[9] P. A. Sturrock, Astrophys. J. 164, 529 (1971).

[10] J. Arons and E. T. Scharlemann, Astrophys. J. 231, 854 (1979).

[11] F. C. Michel, Rev. Mod. Phys. 54, 1 (1982).

[12] F. C. Michel, Theory of Neutron Star Magnetospheres (University of Chicago Press, Chicago, 1991).

[13] E. P. Liang, S. C. Wilks, and M. Tabak, Phys. Rev. Lett. 81, 4887 (1998).

[14] C. Gahn et al., Appl. Phys. Lett. 77, 2662 (2000).

[15] S. C. Wilks et al., Astrophys. Space Sci. 298, 347 (2005).

[16] H. Chen et al., Phys. Rev. Lett. 102, 105001 (2009).

[17] E. M. Campbell and W. J. Hogan, Plasma Phys. Controlled Fusion 41, B39 (1999).

[18] P. Helander and D. J. Ward, Phys. Rev. Lett. 90, 135004 (2003).

[19] C. M. Surko, M. Leventhal, and A. Passner, Phys. Rev. Lett. 62, 901 (1989).

[20] R. G. Greaves, M. D. Tinkle, and C. M. Surko, Phys. Plasmas 1, 1439 (1994).

[21] C. M. Surko and R. G. Greaves, Phys. Plasmas 11, 2333 (2004).
[22] N. Iwamoto, Phys. Rev. E 47, 604 (1993).

[23] G. P. Zank and R. G. Greaves, Phys. Rev. E 51, 6079 (1995).

[24] L. N. Tsintsadze and V. I. Berezhiani, Plasma Phys. Rep. 19, 132 (1993).

[25] P. K. Shukla and L. Stenflo, in Pulsars: Problems and Progress, ASP Conference Series, edited by S. Johnson, M. A. Walker, and M. Bailes (ASP, San Francisco, 1996), p. 171.

[26] T. Cattert, I. Kourakis, and P. K. Shukla, Phys. Plasmas 12, 012319 (2005).

[27] J. Sakai and T. Kawata, J. Phys. Soc. Jpn. 49, 753 (1980).

[28] A. B. Mikhailovskii, O. G. Onishchenko, and A. J. Smolyakov, Sov. J. Plasma Phys. 11, 215 (1985).

[29] L. Stenflo, P. K. Shukla, and M. Y. Yu, Astrophys. Space Sci. 117, 303 (1985).

[30] J. Zhao, K. I. Nishikawa, J. I. Sakai, and T. Neubert, Phys. Plasmas 1, 103 (1994).

[31] P. K. Shukla, N. N. Rao, M. Y. Yu, and N. L. Tsintsadze, Phys. Rep. 138, 1 (1986).

[32] J. H. Adlam and J. E. Allen, Philos. Mag. 3, 448 (1958). 